\title{
Specklegramas de fibra óptica analizados mediante procesamiento digital de imágenes
}

\author{
Luis C. Gutiérrez¹, Luis F. Castaño ${ }^{1}$, Jorge A. Gómez ${ }^{1}$, Jairo C. Quijano¹, Jorge A. Herrera-Ramírez ${ }^{1,2}$, \\ Alejandro Hoyos ${ }^{1}$, Luiz C. Da Silva Nunez ${ }^{3}$, Francisco J. Vélez ${ }^{4}$, Victor H. Aristizabal,* \\ ${ }^{1}$ Grupo de investigación de Física Básica y Aplicada, Politécnico Colombiano Jaime Isaza Cadavid, Medellín, Colombia. \\ ${ }^{2}$ Facultad de Ingenierías, Instituto Tecnológico Metropolitano, Medellín, Colombia \\ ${ }^{3}$ Departamento de Ingeniería Mecánica, Universidad Federal Fluminense, São Paulo, Brasil. \\ ${ }^{4}$ Grupo de Investigación TERMOMEC, Universidad Cooperativa de Colombia, Medellín, Colombia
}

\begin{abstract}
Resumen
Los sensores basados en el análisis de la distribución de intensidades del patrón de interferencia modal a la salida de una fibra óptica son conocidos como sensores ópticos basados en specklegramas de fibra óptica (Fiber Specklegram Sensors, FSSs). En este trabajo se muestran los specklegramas, simulados numéricamente mediante el método de los elementos finitos, de una fibra óptica Thorlabs 1550BHP perturbada mecánicamente, y se hace uso de la información global o de regiones del specklegrama mediante el procesamiento digital de imágenes a través de un análisis de correlación. Los resultados muestran como la correlación entre imágenes se puede usar como valor de cuantificación para la medición de fuerzas, y cómo la división del patrón por zonas de interés puede mejorar las características metrológicas del sensor. (C) 2018. Acad. Colomb. Cienc. Ex. Fis. Nat.
\end{abstract}

Palabras clave: Specklegramas de fibra óptica; Perturbaciones físicas; Sensores de fibra óptica; Simulación numérica.

Fiber specklegram sensor analysis by digital image processing

\begin{abstract}
Fiber Specklegram Sensors (FSSs) are sensors based on the analysis of specklegrams, i.e., the intensity distribution of the modal interference pattern at the output of an optical fiber. By using a finite element method, this work shows numerically simulated specklegrams of an optical fiber Thorlabs 1550BHP under a mechanical perturbation. We employ digital image correlation to analyze the behavior of these specklegrams with different applied forces. The image correlation analysis is applied over the whole specklegram or over selected regions. The results show that the correlation between images is a suitable quantifier of the applied force. We also show that the analysis of selected regions improves the metrological parameters of these sensors. (C) 2018. Acad. Colomb. Cienc. Ex. Fis. Nat.
\end{abstract}

Key words: Fiber Specklegram Sensors; Physical Perturbations; Optical Fiber sensors; Numerical simulation.

\section{Introducción}

Cuando la radiación laser es insertada en una fibra óptica multimodo, los efectos de propagación producen a la salida de la fibra un patrón de speckle (moteado), el cual contiene información sobre el estado espacial de la fibra óptica y que puede ser usado en aplicaciones metrológicas (Arístizabal, Vélez, Rueda, Gómez, \& Gómez, 2016; Efendioglu, 2017; Gasvik, 2002; Hung, 1978; R. Jones and C. Wykes, 1989; Saleh \& Teich, 1991; Yu \& Yin, 2002). De esta manera, pequeñas perturbaciones sobre la fibra multi-modo producirán cambios en la distribución espacial del patrón de speckle que pueden ser detectados mediante cambios locales de intensidad o técnicas de correlación (Crammond, Boyd, \& Dulieu-Barton, 2013; Liu \& Wei, 2007; B. Wang, Huang, Guo, \& Yu, 2003). Los sistemas de sensado basados en patrones de speckle producidos en fibras ópticas multimodo son conocidos como Sensores de Specklegramas de Fibra Óptica (FSS por sus siglas en inglés).

Los FSS han sido ampliamente estudiados mediante arreglos holográficos cuyo medio de registro es un material fotorrefractivo (Gmez, Lorduy G., \& Salazar, 2011; Gómez, Lorduy G., \& Salazar, 2011; Gómez \& Salazar, 2012; Wu, Yin, \& Yu, 1991; Yu, Yin, Zhang, \& Guo, 1994; Yu, Zhang, Yin, \& Ruffin, 1995), sin embargo, más recientemente, se ha explorado la importancia de este tipo de sistemas en esquemas no-holográficos, en la medición

\footnotetext{
*Correspondencia:

Victor H. Aristizabal, vharisti@yahoo.com, victor.aristizabalt@campusucc.edu.co

Recibido: 2 de abril de 2018

Aceptado: 16 de mayo de 2018

Editor: Román Castañeda Sepúlveda
} 
de múltiples parámetros de interés en ingeniería (Fujiwara, Marques dos Santos, \& Suzuki, 2017; Fujiwara, Wu, dos Santos, Schenkel, \& Suzuki, 2017; Fujiwara, Wu, \& Suzuki, 2012; Kumar, Varshney, Antony C, \& Sharma, 2003; Li, Cai, Geng, Qu, \& Fang, 2007; Malki, Gafsi, Michel, Labarrère, \& Lecoy, 1996; Rodriguez-Cobo, Lomer, \& Lopez-Higuera, 2015; B. Wang, Guo, Yin, \& Yu, 2004; Y. Wang, et al., 2008; Yu, Wen, Yin, \& Uang, 1993). Principalmente, dos posibilidades han sido reportadas alrededor de los arreglos no-holográficos. En el primer caso, se han usado herramientas de procesamiento de imágenes para calcular la correlación entre los patrones de speckle perturbados que son capturados mediante cámaras CCD a la salida de la fibra multimodo (Malki, et al., 1996; B. Wang, et al., 2004; Yu, et al., 1993). En el segundo caso, se han usado esquemas experimentales de fibras mono-multi-mono modo para generar y filtrar el patrón de speckle que se genera en la fibra multimodal, lo que permite configurar esquemas de FSS interrogados por variación de potencia óptica (V.H. Aristizabal, Hoyos, Rueda, Gomez, \& Gomez, 2015; Darío Gómez \& Gómez, 2013). Esta estrategia presenta ventajas de simplicidad, estabilidad y bajo costo (Zhang \& Ansari, 2006), pero la correlación entre la distribución espacial de los patrones de speckle y el estado de la fibra óptica se pierde, ya que la salida del sensor es puntual.

En este trabajo exploramos numéricamente la generación de patrones de speckle a la salida de una fibra óptica y el uso del cálculo de valores de correlación entre los patrones de speckle para la medición de perturbaciones físicas, específicamente, esfuerzos mecánicos.

\section{Análisis teórico}

En aproximación de campo cercano, la distribución espacial de intensidad del campo a la salida de una fibra óptica multimodo se puede escribir en coordenadas polares en el punto como (Darío Gómez \& Gómez, 2013):

$$
\begin{aligned}
I=\frac{1}{2} & Y \sum_{m=1}^{M} A_{m}^{2} B_{n m}^{2}\left(U_{m} R\right) \cos ^{2}\left(n_{m} \theta\right) \\
& +\sum_{m=1}^{M} \sum_{l=m+1}^{M} A_{m} A_{l} B_{n m}\left(U_{m} R\right) B_{n l}\left(U_{l} R\right) \cos \left(n_{m} \theta\right) \cos \left(n_{l} \theta\right) \\
& \times \cos \left(\Delta \beta_{m l} z-\Delta \phi_{m l}\right),
\end{aligned}
$$

donde $\Delta \beta_{m l}=\beta_{m}-\beta_{l}$ es la diferencia de las constantes de propagación, $\Delta \phi_{m l}=\phi_{m}-\phi_{l}$ es la diferencia de las constantes de fase, $A_{m}$ y $A_{l}$ son las amplitudes de los modos $m$ y $l$, respectivamente. $R=a / r$ es el radio normalizado siendo $a$ el radio del núcleo de la fibra óptica, $Y=n \sqrt{\varepsilon_{0} / \mu_{0}}$ es la admitancia característica del núcleo donde $n$ es el índice de refracción del núcleo, $U_{m}=a \sqrt{k_{0}^{2} n^{2}-\beta_{m}^{2}}$ es el parámetro modal, $k_{0}=2 \pi \lambda_{0}$ es el número de onda de la luz en el vacío, $\lambda_{0}$ es la longitud de onda en el vacío y $\mathrm{B}_{n m}\left(\mathrm{U}_{m} \mathrm{R}\right)$ es la función de Bessel de primera especie.

Cuando la fibra es perturbada, se asume que los cambios en el patrón de speckle se deben principalmente a cambios de fase y que la intensidad total del patrón de speckle en la fibra es constante (Darío Gómez \& Gómez, 2013). Ahora, teniendo en cuenta lo dicho anteriormente y considerando que al sensor de specklegramas a fibra o FSS (Fiber Specklegram Sensor) se le aplica una perturbación en una pequeña región de longitud $L$, los cambios de fase entre el modo $m$ y $l$ se pueden escribir como $\delta\left(\Delta \beta_{m l} L\right)$. De esta manera, si las variaciones de fase son pequeñas, la nueva distribución de intensidad del patrón a la salida de la fibra multimodo en el punto $(r, \theta)$ es:

$$
\begin{aligned}
I^{\prime}= & \frac{1}{2} Y \sum_{m=1}^{M} A_{m}^{2} B_{n m}^{2}\left(U_{m} R\right) \cos ^{2}\left(n_{m} \theta\right) \\
& +\sum_{m=1}^{M} \sum_{\substack{l=m+1\\
}}^{M} A_{m} A_{l} B_{n m}\left(U_{m} R\right) B_{n l}\left(U_{l} R\right) \cos \left(n_{m} \theta\right) \cos \left(n_{l} \theta\right) \\
& \times \cos \left(\Delta \beta_{m l} z-\Delta \phi_{m l}\right) \pm \delta\left(\Delta \beta_{m l} L\right) \operatorname{sen}\left(\Delta \beta_{m l} z-\Delta \phi_{m l}\right) .
\end{aligned}
$$

Entonces, para calcular los cambios en el patrón de intensidad $\Delta I$ se usan las ecuaciones (1) y (2):

$$
\begin{gathered}
\Delta I=I-I^{\prime}=\sum_{\substack{m=1\\
}}^{M} \sum_{l=m+1}^{M} A_{m} A_{l} B_{n m}\left(U_{m} R\right) B_{n l}\left(U_{l} R\right) \cos \left(n_{m} \theta\right) \cos \left(n_{l} \theta\right) \\
\times\left(\Delta \beta_{m l} L\right) \operatorname{sen}\left(\Delta \beta_{m l} z-\Delta \phi_{m l}\right) .
\end{gathered}
$$

De (3) se puede concluir que las variaciones en el patrón de intensidad son debidos a las diferencias de fase de los modos $m$ y $l$. Entonces, los cambios de fase se pueden escribir como $\Delta \beta_{m l}=n D_{m l}$, donde $D_{m l}$ es una constante proporcional asociada con el cambio de fase entre los modos $m$ y $l$. De esta forma, los cambio en la constante de propagación en la dirección $i(i=x, y o z)$ debido a una perturbación en un segmento $L$ sobre la fibra se puede expresar como

$$
\delta_{i}\left(\Delta \beta_{m l} L\right)=D_{m l} \delta_{i}(n L)
$$

En la ecuación (4) se puede observar que los cambios en las constantes de propagación de los modos que viajan a través de la fibra se deben a cambios en la longitud de camino óptico $\delta_{i}(n L)$. Es posible demostrar que cuando una perturbación es aplicada al segmento de fibra multimodo $\delta_{i}(n L)$ está dado por

$$
\delta_{i}(n L)=\delta_{i} n L+n \delta_{i} L=L\left(\Delta n_{i}+n S_{i}\right)
$$

donde, $\Delta n_{i}=n_{i}-n_{0} \approx-\frac{n_{0}^{3}}{2} P_{i k} S_{k}=-C_{i k} \sigma_{k}$ (aquí se indica en notación tensorial) es el cambio del índice de refracción siendo el material isotrópico en un momento inicial con índice $n_{0}, S_{i}$ es la deformación mecánica (mechanical strain en inglés) y $\sigma_{i}$ es la tensión o esfuerzo mecánico (mechanical stress en inglés) en la dirección principal $i . P_{i k}$ es el tensor de deformación mecánica y $C_{i k}$ es el tensor de esfuerzo mecánico (Arístizabal, et al., 2016). En forma más simplificada:

$$
\begin{aligned}
& \delta_{x}(n L)=L\left(\Delta n_{x}+n S_{x}\right) \\
& \delta_{y}(n L)=L\left(\Delta n_{y}+n S_{y}\right) \\
& \delta_{z}(n L)=L\left(\Delta n_{z}+n S_{z}\right)
\end{aligned}
$$

donde bajo la suposición de deformación plana (plane strain approximation en inglés), es decir $S_{z}=0$ se tiene que los cambios de índice de refracción debido a efectos mecánicos son (Arístizabal, et al., 2016; Mase \& Mase, 1999): 


$$
\begin{gathered}
\Delta n_{x}=n_{x}-n_{0}=-\frac{n_{0}^{3}}{2}\left(P_{11} S_{x}+P_{12} S_{y}\right) \\
\Delta n_{y}=n_{y}-n_{0}=-\frac{n_{0}^{3}}{2}\left(P_{12} S_{x}+P_{11} S_{y}\right) \\
\Delta n_{z}=n_{z}-n_{0}=-\frac{n_{0}^{3}}{2} P_{12}\left(S_{x}+S_{y}\right),
\end{gathered}
$$

donde $P_{11}$ y $P_{12}$ son conocidos como coeficientes Pockels. Entonces, las ecuaciones en (6) quedan

$$
\begin{gathered}
\delta_{x}(n L) \approx n_{0} L\left[S_{x}-\frac{n_{0}^{2}}{2}\left(P_{11} S_{x}+P_{12} S_{y}\right)\right] \\
\delta_{y}(n L) \approx n_{0} L\left[S_{y}-\frac{n_{0}^{2}}{2}\left(P_{12} S_{x}+P_{11} S_{y}\right)\right] \\
\delta_{z}(n L) \approx n_{0} L\left[S_{z}-\frac{n_{0}^{2}}{2} P_{12}\left(S_{x}+S_{y}\right)\right]
\end{gathered}
$$

En las ecuaciones (8), si las deformaciones mecánicas son pequeñas del orden de $10^{-3}$ en fibras ópticas (Gianino \& Bendow, 1981), se puede despreciar el término que contiene los coeficientes Pockels debido a que estos son mucho menores que uno (Arístizabal, et al., 2016; Victor H. Aristizabal, Velez, \& Torres, 2006), obteniéndose la ecuación simplificada:

$$
\delta(n L)=\delta_{x}(n L)=\delta_{y}(n L)=\delta_{z}(n L) \approx n_{0} L S_{z} .
$$

En la ecuación aproximada (9), $S_{z}$ se puede estimar como la deformación unitaria de ingeniería que se define como $\left(L-L_{0}\right) / L_{0}$. Al reemplazar la ecuación (9) en (3), se puede observar que los cambios de la intensidad en el patrón de speckle varía linealmente con la deformación mecánica producida en la fibra óptica. En conclusión, cuando la fibra óptica es perturbada, el patrón de speckle a la salida de la fibra es ligeramente modificado por los cambios de fase en la fibra óptica multimodo. Estas modificaciones producen variaciones locales de intensidad lo que genera pequeñas deformaciones en el patrón de speckle de la fibra. Este tipo de comportamiento se ilustra en (Arístizabal, et al., 2016) donde la intensidad es perturbada localmente deformando ligeramente el patrón de speckle de la fibra para el caso de una perturbación mecánica.

En este trabajo se empleará un método alternativo para calcular las intensidades definidas en las expresiones (1) y (2). Primero, para calcular las distribuciones de intensidad del campo eléctrico para una fibra multimodo bajo la aplicación de una línea de carga mecánica (Arístizabal, et al., 2016; Victor H. Aristizabal, et al., 2006), se soluciona numéricamente mediante el método de los elementos finitos la ecuación de onda vectorial para una onda monocromática (V.H. Aristizabal, Vélez, \& Torres, 2004, 2006; Torres, Aristizábal, \& Andrés, 2011):

$$
\nabla \times \nabla \times \overrightarrow{\mathrm{E}}-k_{0}^{2} n^{2} \overrightarrow{\mathrm{E}}=0,
$$

dónde $n$ es la distribución del índice de refracción de la fibra y se puede expresar como:

$$
n=\left[\begin{array}{ccc}
n_{x} & 0 & 0 \\
0 & n_{y} & 0 \\
0 & 0 & n_{z}
\end{array}\right]
$$

siendo $n_{x}, n_{y}$ y $n_{z}$ los índices definidos en (7). $n_{0}$ para el núcleo se calculó usando la ecuación de Sellmeier para el Sílice fundido (Arístizabal, et al., 2016):

$$
\begin{aligned}
n_{\text {onúcleo }}^{2}(\lambda[\mu \mathrm{m}])= & 1+\frac{0.6961663 \lambda_{0}{ }^{2}}{\lambda_{0}{ }^{2}-0.0684043^{2}} \\
& +\frac{0.4079426 \lambda_{0}{ }^{2}}{\lambda_{0}{ }^{2}-0.1162414^{2}}+\frac{0.8974794 \lambda_{0}{ }^{2}}{\lambda_{0}{ }^{2}-9.896161^{2}}
\end{aligned}
$$

y $n_{0}$ para el revestimiento se computó desde la ecuación (12) y la apertura numérica de la fibra bajo estudio $(N A=$ $\left.\sqrt{n_{\text {onúcleo }}^{2}-n_{\text {orevestimiento }}^{2}}\right)$. De esta manera se obtienen el campo vectorial de los modos y las constantes de propagación para el sistema perturbado mecánicamente. Todos los modos calculados se suman vectorialmente para hallar la intensidad del campo resultante, obteniéndose finalmente el patrón de speckle (Arístizabal, et al., 2016).

Ya que los cambios de intensidad por perturbaciones se dan a una escala local cercana a la del tamaño del grano de speckle, estos cambios no se pueden cuantificar adecuadamente por medio del promedio de la intensidad del patrón speckle completo. Así, cuando se tienen imágenes, regularmente se usan técnicas de correlación para esta cuantificación. La implementación algebraica de esta técnica se puede llevar a cabo por medio de varias expresiones similares. (Efendioglu, 2017; Gubarev, Li, Klenovskii, \& Glotov, 2016), aunque la más común es el coeficiente de correlación $(C)$ dado por:

$$
C=\frac{\sum_{i} \sum_{j}\left(\left(I_{r e f}(i, j)-\left\langle I_{r e f}\right\rangle\right) \cdot\left(I_{n}(i, j)-\left\langle I_{n}\right\rangle\right)\right)}{\sqrt{\left(\sum_{i} \sum_{j}\left(I_{r e f}(i, j)-\left\langle I_{r e f}\right\rangle\right)^{2}\right)\left(\sum_{i} \sum_{j}\left(I_{n}(i, j)-\left\langle I_{n}\right\rangle\right)^{2}\right)}}
$$

donde $\left\langle I_{r e f}\right\rangle$ y $\left\langle I_{n}\right\rangle$ corresponden a las intensidades promedio del patrón de speckle a la salida de la fibra en un estado de referencia y perturbada, respectivamente.

\section{Resultados y discusión}

Siguiendo la metodología explicada anteriormente, se calculó y simuló el patrón de speckle para una fibra multimodo (MMF) Thorlabs 1550BHP, con los parámetros descritos en la Tabla 1, donde la fuente de luz tiene una distribución gaussiana y el campo eléctrico polarizado en $x$. A la fibra multimodo se le aplicó una fuerza en dirección contraria a $y$. En la Figura 1 se ilustra la situación física que se simula. Los patrones de la Figura 2 muestran el speckle obtenido mediante la superposición de pocos modos para diferentes valores de la fuerza de perturbación, alrededor de 16 modos que son los que se propagan en la fibra estudiada. En este caso, debido a que la fibra que estudiamos sólo soporta 16 modos de propagación, la distribución de puntos brillantes y oscuros no corresponde a la de un patrón de speckle bien comportado, sino una distribución de intensidades con una modulación más suave extendida sobre toda la salida de la 
fibra. Así, estas imágenes hacen evidente la interferencia entre los modos de propagación y el intercambio de energía entre ellos al perturbar la fibra.

Al analizar la evolución de estos patrones completos usando la medida de correlación entre ellos en diferentes estados de perturbación, se encuentran varios comportamientos

Tabla 1. Propiedades de la fibra óptica usada en la simulación con una apertura numérica de 0.13

\begin{tabular}{lcc}
\hline Parámetro & Núcleo & Revestimiento \\
\hline Diámetro $[\mu \mathrm{m}]$ & 9,0 & 125 \\
Densidad $\left[\mathrm{kg} / \mathrm{m}^{3}\right]$ & 2201 & 2201 \\
Módulo de Young $[\mathrm{GPa}]$ & 72,6 & 72,6 \\
Coeficiente de Poisson & 0,164 & 0,164 \\
Primer coeficiente de Pockels: $P_{11}$ & 0,121 & 0,121 \\
Segundo coeficiente de Pockels: $P_{12}$ & 0,270 & 0,270 \\
$\begin{array}{l}\text { Índice de refracción antes de aplicar } \\
\text { la fuerza externa: } n_{0} \text { a } 632,8 \mathrm{~nm}\end{array}$ & 1,4570 & 1,4521 \\
\hline
\end{tabular}

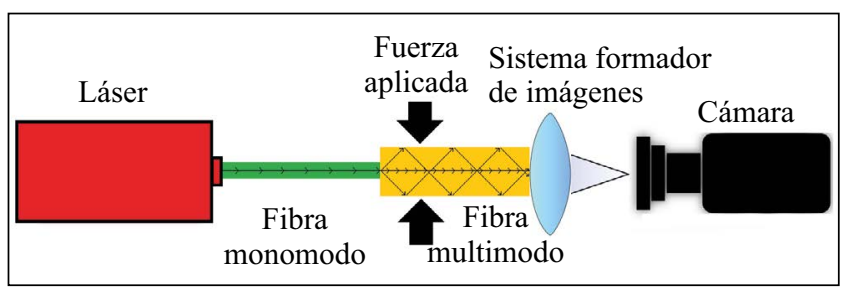

Figura 1. Diagrama del escenario físico que recrea la simulación. para los diferentes rangos de fuerza por unidad de longitud aplicada. El coeficiente de correlación se comporta linealmente para ciertos rangos, como lo muestra la Figura $3 \mathrm{~b}$ y $3 \mathrm{c}$, pero en otros no existe un comportamiento lineal, ni siquiera monotónico (Figura 3a y 3d). Adicionalmente, la sensibilidad también es dependiente del rango de fuerzas analizado, por ejemplo, la sensibilidad es más alta en la Figura $3 c$ que en la Figura $3 b$ o $3 d$, si en esta última se redujera el rango de trabajo a la zona lineal de la curva.

La medida de correlación es dependiente de que tan grandes son los cambios en la distribución de intensidades del patrón al cambiar la fuerza aplicada. Ante cambios grandes en la intensidad del patrón se pueden generar comportamientos no monotónicos como de discontinuidad de las curvas de correlación. Una propuesta para evitar estos inconvenientes es la redefinición del patrón de intensidades usado como referencia en los cálculos de correlación, lo que equivale a una recalibración del sistema, pero también es algo que permite aprovechar zonas específicas dónde el comportamiento de la curva de correlación sea el adecuado. En la Figura $3 b$ se muestra la curva de correlación en la que el patrón de referencia usado es el proveniente de la fuerza por unidad de longitud de $4 \mathrm{~N} / \mathrm{mm}$ y con respecto a este patrón es que se calculan las correlaciones de los demás puntos en esa figura.

Si se hace una división en regiones del patrón de intensidades, como por ejemplo la región del rectángulo rojo en la figura 4a, y se analiza la correlación para esta región en diferentes patrones de intensidad (diferentes fuerzas aplicadas), se puede mejorar el comportamiento de las curvas de respuesta en correlación. De esta manera, se puede
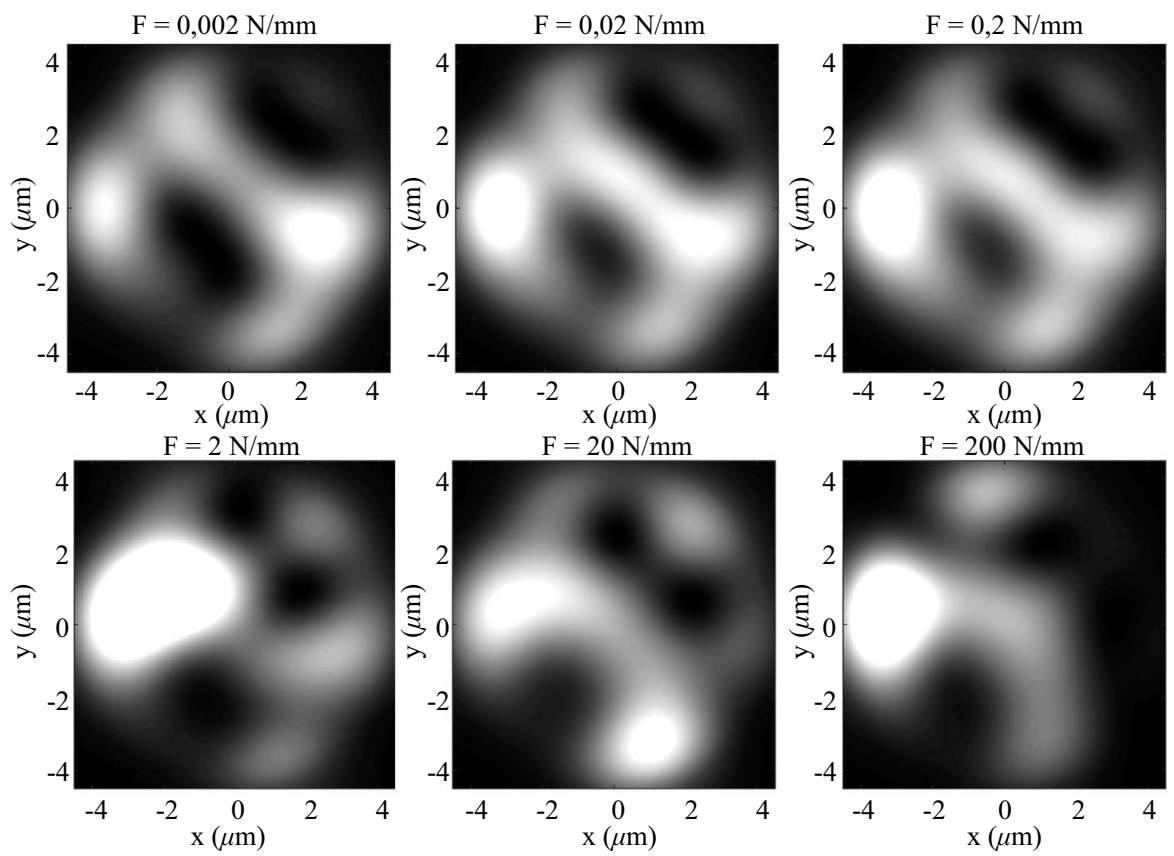

Figura 2. Imágenes de patrones de speckle en una fibra multimodo simulados numéricamente a $650 \mathrm{~nm}$ para varios valores de fuerza por unidad de longitud aplicada. 

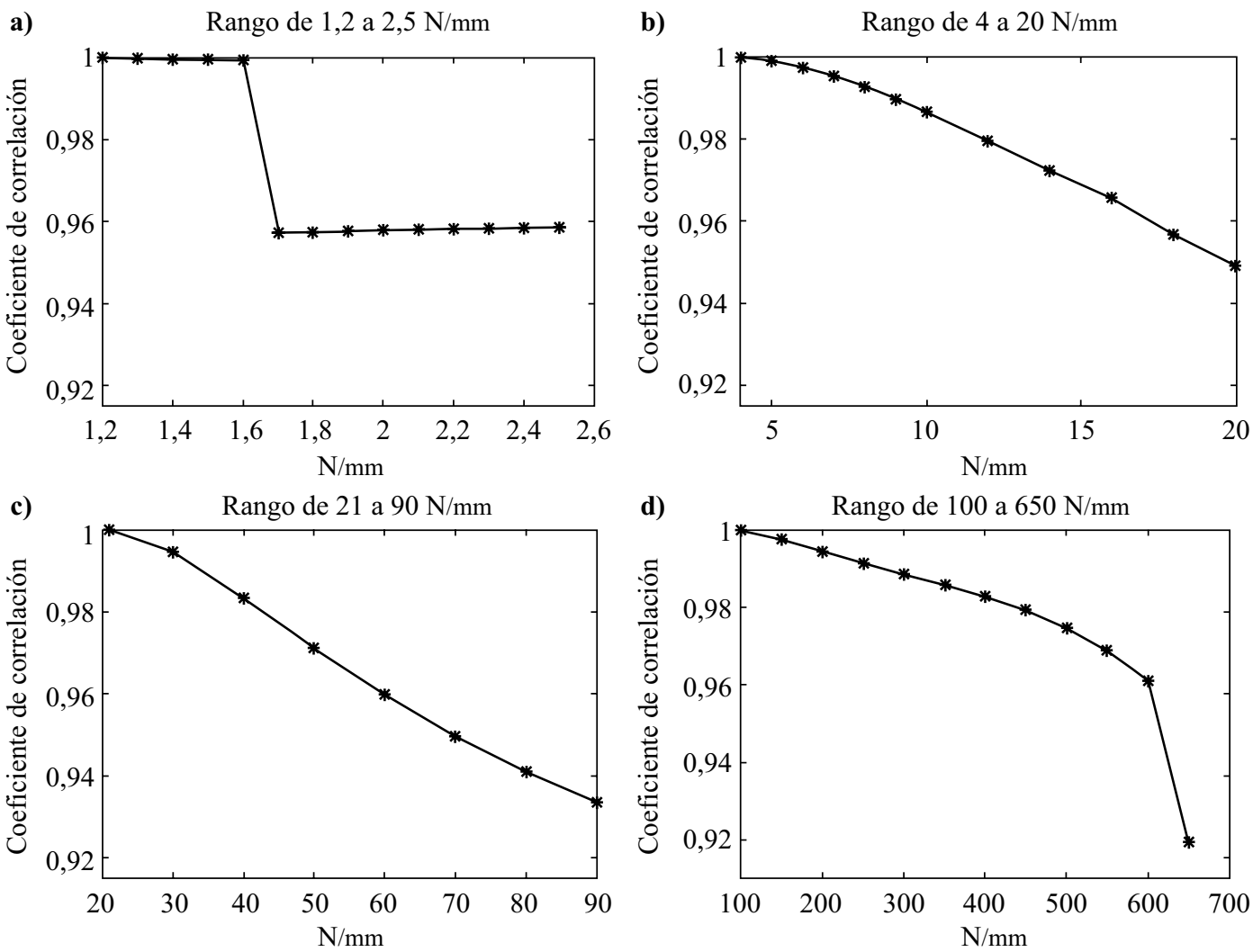

Figura 3. Curvas de correlación entre patrones de speckle a la salida de la fibra para diferentes rangos de fuerza por unidad de longitud aplicada.
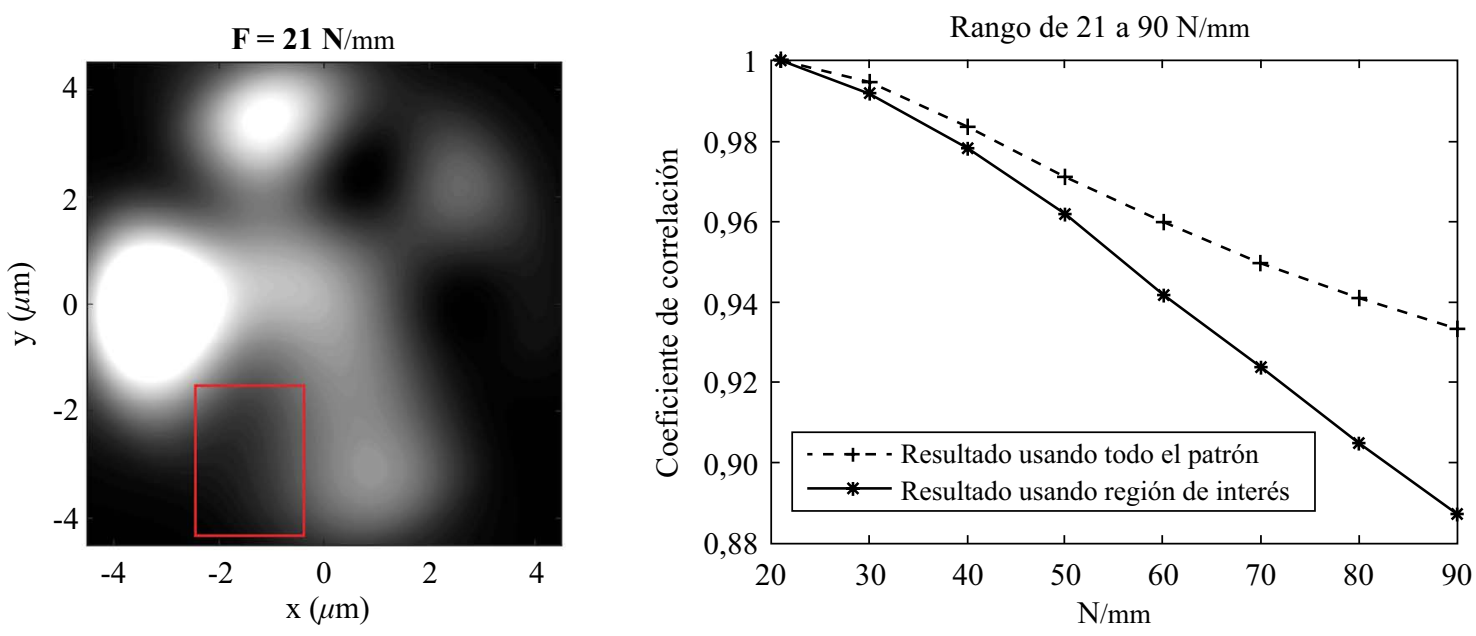

Figura 4. División del patrón de intensidades para la medida de correlación. a) Patrón de intensidades completo y zona de división o interés, rectángulo rojo. b) comparación de curva de correlación usando todo el patrón o una zona de interés menor.

mejorar en la capacidad de sensado del sistema según sea el requerimiento. La figura $4 \mathrm{~b}$ muestra la curva de correlación para el rango de fuerzas entre $21 \mathrm{~N} / \mathrm{mm}$ y $90 \mathrm{~N} / \mathrm{mm}$ (antes mostrado en la figura 3c), usando la zona del rectángulo rojo mencionado, el cual tiene una mayor sensibilidad manteniendo una alta linealidad. Este caso muestra las posibilidades de mejorar las características metrológicas del sensor al usar diferentes zonas del patrón de salida. Finalmente, se puede observar que la sensibilidad y el rango dinámico dependerán de cómo cambia el patrón de intensidades para cada zona elegida. Así, se pueden encontrar zonas que mejoren ostensiblemente el desempeño del sensor como otras que producen comportamientos no monotónicos o discontinuidades en un rango de fuerza requerido. 


\section{Conclusiones}

En este trabajo se llevó a cabo la simulación numérica de la acción de una fuerza de perturbación sobre una fibra óptica y la respuesta en el patrón de intensidades a la salida de ésta. Los resultados son cercanos a los observados experimentalmente y mostrados en la literatura. Sobre estos patrones se realizó análisis de correlación como método de cuantificación/interrogación del sistema al ser usado como sensor de perturbaciones físicas. Se mostró que esta metodología es útil y que la división del patrón de intensidades puede mejorar las características metrológicas del sistema como sensor.

\section{Agradecimientos}

Los autores agradecen al Politécnico Colombiano Jaime Isaza Cadavid, a la Universidad Cooperativa de Colombia y a la Universidade Federal Fluminense. V.H. Aristizabal y F.J. Velez agradecen el apoyo financiero de la Universidad Cooperativa de Colombia mediante el proyecto con código INV1898.

\section{Contribución de los autores}

los autores mencionados anteriormente han contribuido de manera original y eficiente para el desarrollo completo del artículo.

El trabajo fue presentado en la modalidad de póster en el XV Encuentro Nacional de Óptica y VI Conferencia Andina y del Caribe en Óptica y sus aplicaciones (ENO-CANCOA), evento llevado a cabo en la Universidad Industrial de Santander sede Bucarica, en Bucaramanga, Colombia del 20 al 24 de noviembre de 2017.

\section{Conflicto de intereses}

Los autores y coautores de este trabajo no tienen ningún conflicto de intereses de índole académico, personal, religioso, ético y profesional.

\section{Referencias}

Aristizabal, V. H., Hoyos, A., Rueda, E., Gomez, N. D., \& Gomez, J. A. (2015). Effect of wavelength on metrological characteristics of non-holographic fiber specklegram sensor. Photonic Sensors, 5 (1). http://doi.org/10.1007/ s13320-014-0210-3

Arístizabal, V. H., Vélez, F. J., Rueda, E., Gómez, N. D., \& Gómez, J. A. (2016). Numerical modeling of fiber specklegram sensors by using finite element method (FEM). Optics Express, 24 (24): 27225-27238. http://doi. org/10.1364/OE.24.027225

Aristizabal, V. H., Velez, F. J., \& Torres, P. (2006). Numerical model and analysis of optical fibers with internal electrodes. Revista Colombiana de Física. 38 (1): 173-176. Retrieved from http://revcolfis.org/publicaciones/vol38_1/ resumenes/3801173.htm

Aristizabal, V. H., Vélez, F. J., \& Torres, P. (2004). Modeling of photonic crystal fibers with the Scalar Finite Element Method. In Proceedings of SPIE - The International Society for Optical Engineering (Vol. 5622). http://doi. org/10.1117/12.591051
Aristizabal, V. H., Vélez, F. J., \& Torres, P. (2006). Analysis of photonic crystal fibers: Scalar solution and polarization correction. Optics Express. 14 (24). http://doi.org/10.1364/ OE.14.011848

Crammond, G., Boyd, S. W., \& Dulieu-Barton, J. M. (2013). Speckle pattern quality assessment for digital image correlation. Optics and Lasers in Engineering. 51 (12): 1368-1378. http://doi.org/10.1016/j.optlaseng.2013.03.014

Darío Gómez, N., \& Gómez, J. A. (2013). Effects of the speckle size on non-holographic fiber specklegram sensors. Optics and Lasers in Engineering. 51 (11): 1291-1295. http://doi. org/10.1016/j.optlaseng.2013.05.007

Efendioglu, H. S. (2017). A Review of Fiber-Optic Modal Modulated Sensors: Specklegram and Modal Power Distribution Sensing. IEEE Sensors Journal. 17 (7): 2055-2064. http://doi.org/10.1109/JSEN.2017.2658683

Fujiwara, E., Marques dos Santos, M. F., \& Suzuki, C. K. (2017). Optical fiber specklegram sensor analysis by speckle pattern division. Applied Optics. 56 (6): 1585. http://doi.org/10.1364/AO.56.001585

Fujiwara, E., Wu, Y. T., dos Santos, M. F. M., Schenkel, E. A., \& Suzuki, C. K. (2017). Development of a tactile sensor based on optical fiber specklegram analysis and sensor data fusion technique. Sensors and Actuators A: Physical. 263: 677-686. http://doi.org/10.1016/j.sna.2017.07.031

Fujiwara, E., Wu, Y. T., \& Suzuki, C. K. (2012). Vibration-based specklegram fiber sensor for measurement of properties of liquids. Optics and Lasers in Engineering. 50 (12): 17261730. http://doi.org/10.1016/j.optlaseng.2012.06.018

Gasvik, K. J. (2002). Optical Metrology (3rd ed.). Chichester, England: John Wiley \& Sons Ltd.

Gianino, P. D., \& Bendow, B. (1981). Calculations of stressinduced changes in the transverse refractive-index profile of optical fibers. Applied Optics. 20 (3): 430. http://doi. org/10.1364/AO.20.000430

Gómez, J. A., Lorduy G., H., \& Salazar, Á. (2011). Improvement of the dynamic range of a fiber speckleg\ram sensor based on volume speckle recording in photorefractive materials. Optics and Lasers in Engineering. 49 (3): 473-480. http:// doi.org/10.1016/j.optlaseng.2010.11.017

Gómez, J. A., Lorduy G., H., \& Salazar, Á. (2011). Influence of the volume speckle on fiber specklegram sensors based on four-wave mixing in photorefractive materials. Optics Communications. 284 (4): 1008-1014. http://doi. org/10.1016/j.optcom.2010.10.037

Gómez, J. A., \& Salazar, Á. (2012). Self-correlation fiber specklegram sensor using volume characteristics of speckle patterns. Optics and Lasers in Engineering. 50 (5): 812-815. http:// doi.org/10.1016/j.optlaseng.2012.01.002

Gubarev, F., Li, L., Klenovskii, M., \& Glotov, A. (2016). Speckle pattern processing by digital image correlation. MATEC Web of Conferences. 48: 4003. http://doi.org/10.1051/ matecconf/20164804003

Hung, Y. Y. (1978). Displacement and strain measurement. In R. K. Erf (Ed.), Speckle metrology (pp. 51-71). New York: Academic Press, Inc.

Kumar, A., Varshney, R. K., Antony C, S., \& Sharma, P. (2003). Transmission characteristics of SMS fiber optic sensor structures. Optics Communications. 219 (1-6): 215-219. http://doi.org/10.1016/S0030-4018(03)01289-6 
Li, J., Cai, H., Geng, J., Qu, R., \& Fang, Z. (2007). Specklegram in a multiple-mode fiber and its dependence on longitudinal modes of the laser source. Applied Optics. 46 (17): 3572. http://doi.org/10.1364/AO.46.003572

Liu, Y., \& Wei, L. (2007). Low-cost high-sensitivity strain and temperature sensing using graded-index multimode fibers. Applied Optics. 46 (13): 2516-2519. http://doi.org/10.1364/ AO.46.002516

Malki, A., Gafsi, R., Michel, L., Labarrère, M., \& Lecoy, P. (1996). Impact and vibration detection in composite materials by using intermodal interference in multimode optical fibers. Applied Optics. 35 (25): 5198. http://doi. org/10.1364/AO.35.005198

Mase, G. T., \& Mase, G. E. (1999). Continuum for Engineers. New York (2 Ed). Boca Raton: CRC Press.

R. Jones and C. Wykes. (1989). Holographic and Speckle Interferometry. Cambridge University Press. http://doi. org/10.1017/CBO9780511622465

Rodriguez-Cobo, L., Lomer, M., \& Lopez-Higuera, J.-M. (2015). Fiber Specklegram-Multiplexed Sensor. Journal of Lightwave Technology. 33 (12): 2591-2597. http://doi. org/10.1109/JLT.2014.2364318

Saleh, B. E. a, \& Teich, M. C. (1991). Fundamentals of Photonics (Vol. 5). New York, USA: John Wiley \& Sons, Inc. http:// doi.org/10.1002/0471213748

Torres, P., Aristizábal, V. H., \& Andrés, M. V. (2011). Modeling of photonic crystal fibers from the scalar wave equation with a purely transverse linearly polarized vector potential. Journal of the Optical Society of America B: Optical Physics. 28 (4). http://doi.org/10.1364/JOSAB.28.000787

Wang, B., Guo, R., Yin, S., \& Yu, F. T. S. (2004). Chemical Sensing with Hetero-Core Fiber Specklegram. Journal of Holography and Speckle. 1 (1): 53-57. http://doi.org/10. 1166/jhs. 2004.008
Wang, B., Huang, C., Guo, R., \& Yu, F. T. S. (2003). A novel fiber chemical sensor using inner-product multimode fiber speckle fields. In F. T. S. Yu, R. Guo, \& S. Yin (Eds.), Proceedings of SPIE - The International Society for Optical Engineering (p. 299). http://doi.org/10.1117/12.515977

Wang, Y., Cai, H., Qu, R., Fang, Z., Marin, E., \& Meunier, J.-P. (2008). Specklegram in a grapefruit fiber and its response to external mechanical disturbance in a single-multiple-single mode fiber structure. Applied Optics. 47 (20): 3543. http:// doi.org/10.1364/AO.47.003543

Wu, S., Yin, S., \& Yu, F. T. S. (1991). Sensing with fiber specklegrams. Applied Optics. 30 (31): 4468. http://doi. org/10.1364/AO.30.004468

Yu, F. T. S., Wen, M., Yin, S., \& Uang, C.-M. (1993). Submicrometer displacement sensing using inner-product multimode fiber speckle fields. Applied Optics. 32 (25): 4685. http:// doi.org/10.1364/AO.32.004685

Yu, F. T. S., \& Yin, S. (2002). Fiber Optic Sensors. New York: Marcel Dekker, Inc.

Yu, F. T. S., Yin, S., Zhang, J., \& Guo, R. (1994). Application of a fiber-speckle hologram to fiber sensing. Applied Optics. 33 (22): 5202. http://doi.org/10.1364/AO.33.005202

Yu, F. T. S., Zhang, J., Yin, S., \& Ruffin, P. B. (1995). Analysis of a fiber specklegram sensor by using coupled-mode theory. Applied Optics. 34 (16): 3018. http://doi.org/10.1364/AO. 34.003018

Zhang, Z., \& Ansari, F. (2006). Fiber-optic laser speckleintensity crack sensor for embedment in concrete. Sensors and Actuators A: Physical. 126 (1): 107-111. http://doi. org/10.1016/j.sna.2005.10.002 